\title{
The structural determinants of insulin-like peptide 3 activity
}

\author{
${ }^{1}$ Florey Neuroscience Institutes, University of Melbourne, Melbourne, VIC, Australia \\ ${ }^{2}$ Department of Biochemistry and Molecular Biology, University of Melbourne, Melbourne, VIC, Australia \\ ${ }^{3}$ Department of Pharmacology, University of Melbourne, Melbourne, VIC, Australia \\ ${ }^{4}$ School of Biomedical Sciences, The University of Queensland, Brisbane, QLD, Australia \\ ${ }^{5}$ School of Chemistry, University of Melbourne, Melbourne, VIC, Australia
}

Ross A. D. Bathgate ${ }^{1,2}{ }^{*}$, Soude Zhang ${ }^{1}$, Richard A. Hughes ${ }^{3}$, K. Johan Rosengren ${ }^{4}$ and John D. Wade ${ }^{1,5 *}$

Edited by:

Kenneth Siddle, University of

Cambridge, UK

Reviewed by:

Andrzej Marek Brzozowski, University of York, UK

Chengcheng Alec Zhang, UT

Southwestern Medical Center, USA

${ }^{*}$ Correspondence:

Ross A. D. Bathgate and

John D. Wade, Florey Neuroscience Institutes, University of Melbourne,

Melbourne, VIC 3031, Australia.

e-mail: bathgate@florey.edu.au;

wade@florey.edu.au
Insulin-like peptide 3 (INSL3) is a hormone and/or paracrine factor which is a member of the relaxin peptide family. It has key roles as a fertility regulator in both males and females. The receptor for INSL3 is the leucine rich repeat (LRR) containing G-protein coupled receptor 8 (LGR8) which is now known as relaxin family peptide receptor 2 (RXFP2). Receptor activation by INSL3 involves binding to the LRRs in the large ectodomain of RXFP2 by residues within the B-chain of INSL3 as well as an interaction with the transmembrane exoloops of the receptor. Although the binding to the LRRs is well characterized the features of the peptide and receptor involved in the exoloop interaction are currently unknown. This study was designed to determine the key INSL3 determinants for RXFP2 activation. A chimeric peptide approach was first utilized to demonstrate that the A-chain is critical for receptor activation. Replacement of the INSL3 A-chain with that from the related peptides INSL5 and INSL6 resulted in complete loss of activity despite only minor changes in binding affinity. Subsequent replacement of specific A-chain residues with those from the INSL5 peptide highlighted that the N-terminus of the A-chain of INSL3 is critical for its activity. Remarkably, replacement of the entire $\mathrm{N}$-terminus with four or five alanine residues resulted in peptides with near native activity suggesting that specific residues are not necessary for activity. Additionally removal of two amino acids at the $\mathrm{C}$-terminus of the A-chain and mutation of Lys-8 in the B-chain also resulted in minor decreases in peptide activity. Therefore we have demonstrated that the activity of the INSL3 peptide is driven predominantly by residues 5-9 in the A-chain, with minor additional contributions from the two C-terminal A-chain residues and Lys-8 in the B-chain. Using this new knowledge, we were able to produce a truncated INSL3 peptide structure which retained native activity, despite having 14 fewer residues than the parent peptide.

Keywords: relaxin, insulin-like peptide 3, RXFP1, RXFP2, peptides, GPCR

\section{INTRODUCTION}

Insulin-like peptide 3 (INSL3) was originally identified as a novel gene highly expressed in the Leydig cells of the testis, and was initially named Leydig insulin-like peptide (Ley-I-L; Adham et al., 1993). Its homology to the peptide hormone relaxin also led to the name relaxin-like factor (RLF; Bullesbach and Schwabe, 1995). However INSL3 is now its common name and it is known to be a member of the relaxin peptide family. The relaxin peptides are all characterized by a common structure comprising two chains (A and B) that are cross-linked by disulfide bonds (Figure 1). This arrangement results in a general structure in which three helical segments (two in the A-chain and one in the B-chain) enclose a hydrophobic core (Rosengren et al., 2009). Like other members of this family, the native receptor for INSL3 is a G-protein coupled receptor (GPCR; Hsu et al., 2002). INSL3 binds with high affinity to the leucine-rich repeat-containing, $G$ protein coupled receptor 8 (LRG8; Kumagai et al., 2002), now known as the relaxin family peptide receptor 2 (RXFP2; Bathgate et al., 2006a).

Insulin-like peptide 3 is expressed in the Leydig cells of every mammalian species so far investigated (Bathgate et al., 2006b).
It is also expressed in the ovary (Ivell and Bathgate, 2002) where it is localized in the follicular thecal cells and the corpus luteum (Tashima et al., 1995; Bathgate et al., 1996; Bamberger et al., 1999). Studies in INSL3 (Nef and Parada, 1999; Zimmermann et al., 1999) and RXFP2 (Gorlov et al., 2002) knockout male mice have demonstrated that INSL3 acting through its receptor is essential for the trans-abdominal phase of testicular descent where it is necessary for the growth and development of the gubernaculum. Hence, male mice lacking the INSL3 gene are bilaterally cryptorchid. INSL3 is also a circulating hormone in adult males but its precise role is still unknown. Studies in rats suggest that INSL3 may play a role in germ cell survival in adult animals (Kawamura et al., 2004). INSL3 treatment suppressed male germ cell apoptosis induced by gonadotropin in male rats suggesting that INSL3 is a paracrine factor involved in male germ cell survival. In the female a potential role for INSL3 in ovarian physiology was first suggested by the impaired fertility found in female INSL3 knock out mice (Nef and Parada, 1999). Further studies in an independent INSL3 knock out mouse strain (Zimmermann et al., 1999) identified a higher apparent rate of atresia or regression of both follicles and corpora lutea 
A

INSL3

H2 Relaxin

H3 Relaxin

INSL5

INSL6

INSL3

$\mathrm{H} 2$ relaxin

$\mathrm{H} 3$ relaxin

INSL5

INSL6
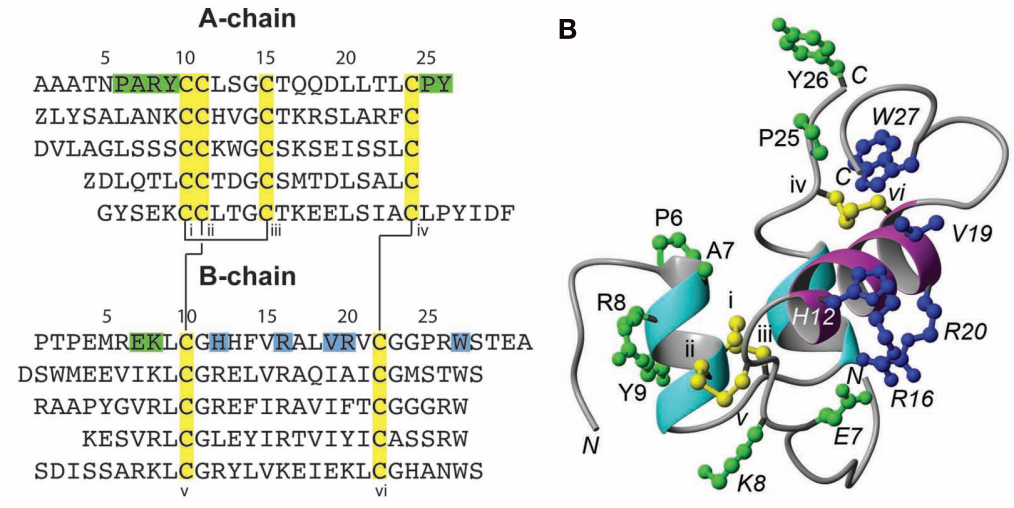

FIGURE 1 | Sequences and three dimensional structure of relaxin peptides. (A) Sequences of the A- and B-chains of INSL3 and selected related members of the relaxin peptide family. (B) Solution NMR structure of INSL3 illustrating the typical overall fold of relaxins. The helical segments of the A-chain are shown in cyan and the single helical segment of the B-chain is shown in magenta. The conserved cysteine residues forming three disulfide bonds are shown in ball-and-stick representation and are highlighted in yellow in both panels and numbered sequentially by roman numbers. The disulfide connectivity is illustrated by connecting lines in (A). Residues in the B-chain that have been shown to be important for receptor binding are highlighted in blue and additional residues whose potential roles in the activation of INSL3 are discussed throughout this paper are highlighted in green. resulting in fewer follicles, corpora lutea, and offspring (SpanelBorowski et al., 2001). These data imply an important role for INSL3 in protecting follicular and luteal cells from entering the standard default pathway of apoptosis and atresia. Further evidence for an anti-apoptotic role for INSL3 comes from studies examining INSL3 expression in bovine follicles (Irving-Rodgers et al., 2002). Studies in rats suggest that INSL3 may also have a role in oocyte development (Kawamura et al., 2004). In vitro and in vivo studies in cultured rat preovulatory follicles indicated that INSL3 treatment initiates meiotic progression of arrested oocytes. Hence the authors suggested that INSL3 may play a role in LHinduced oocyte maturation. Importantly, all these studies suggest that INSL3 has key roles in fertility in both males and females and compounds targeting RXFP2 may be novel fertility regulating agents.

Human RXFP2 can be activated by both INSL3 and, at higher concentrations, relaxin. However the native receptor for relaxin is the closely related receptor RXFP1 (LGR7), for which INSL3 has a very poor affinity. We now have a basic understanding of the structural mechanisms by which relaxin and INSL3 bind to and activate their receptors. The ligands bind to both the leucine rich repeats (LRRs) of the receptor ectodomains and the transmembrane exoloops; the low density lipoprotein Class A (LDLa) domain at the $\mathrm{N}$-terminus of the receptor is necessary for receptor activation (Hartley et al., 2009). Primary ligand binding occurs between residues from the $\mathrm{B}$-chain of the peptide and residues in the inner beta-sheets of the LRRs of the receptor (Hartley et al., 2009). These binding sites are well characterized for both relaxin-RXFP1 (Bullesbach and Schwabe, 2005b) and INSL3RXFP2 (Scott et al., 2007). Current evidence suggests that relaxin binds to the RXFP2 LRRs utilizing a hybrid relaxin/INSL3 binding site comprising some of the INSL3-RXFP2 interactions but also utilizing the partially conserved RXFP1-relaxin binding site in the RXFP2 LRRs (Scott et al., 2009). The peptide binding site in the transmembrane exoloops of the receptors is less well characterized but is believed to involve the A-chain of the peptides, although the residues involved have not been fully determined in either the peptides or the receptors. There is little known about the regions of the relaxin A-chain involved in receptor interaction although there is considerable evidence to suggest that the N-terminus of the INSL3 A-chain is involved in the activation of RXFP2 by INSL3 (Bullesbach and Schwabe, 2005a, 2007). Importantly, unlike the previously characterized INSL3 B-chain residues B12, B16, B19, $\mathrm{B} 20$, and B27 which are involved in binding and activity (Bullesbach and Schwabe, 2005a; Rosengren et al., 2006) the N-terminus of the A-chain does not contribute to the binding affinity. This study set out to determine the key molecular determinants of the INSL3 peptide involved in activation of the RXFP2 receptor.

\section{MATERIALS AND METHODS \\ PEPTIDES}

Recombinant DNA-derived human gene-2 (H2) relaxin was kindly provided by Corthera (San Mateo, CA, USA). Synthetic europiummonolabeled INSL3 and native human INSL3 were prepared inhouse as previously described (Rosengren et al., 2006; Shabanpoor et al., 2008).

\section{SYNTHESIS OF INSL3 PEPTIDES}

Each of the chimeric or mutant INSL3 peptides were prepared by optimized solid phase synthesis of the separate A- and B-chains followed by their combination via regioselective disulfide bond formation as previously described (Zhang et al., 2010). Overall yields of purified peptides averaged $10-14 \%$ based on starting material in the form of cleaved B-chain and each was comprehensively chemically characterized by analytical RP-HPLC and MALDI-TOF mass spectrometry. Amino acid analysis of acid hydrolyzed aliquots enabled peptide quantitation prior to bioassay.

\section{LIGAND BINDING ASSAYS}

HEK-293T cells stably transfected with RXFP2 were seeded in 96 well Optiplates and Eu-INSL3 binding assays conducted as described previously (Shabanpoor et al., 2008). Competition 
binding assays were performed using increasing concentrations of unlabeled INSL3 and non-specific binding was determined using $1 \mu \mathrm{M}$ unlabeled INSL3. Data are expressed as mean \pm SEM of $\%$ specific binding of triplicate measurements pooled from at least three independent experiments. Data were analyzed using GraphPad PRISM (GraphPad Inc) and a non-linear regression one-site binding model was used to plot curves and calculate $\mathrm{pK}_{\mathrm{i}}$ values. Final pooled $\mathrm{pK}_{\mathrm{i}}$ data were analyzed using one-way ANOVA coupled to Newman-Keuls multiple comparison test for multiple group comparison.

\section{CAMP ACTIVITY ASSAYS}

The ability of the INSL3 peptide analogs to stimulate receptor signaling was assessed using a cAMP reporter gene assay (Chen et al., 1995) as previously described (Scott et al., 2006). Briefly, HEK-293T cells were co-transfected with receptor constructs and a pCRE- $\beta$-galactosidase reporter plasmid (Chen et al., 1995) in 96 well plates. The RXFP2/1 construct contains the ectodomain of RXFP2 fused to the transmembrane domains of RXFP1 and has been previously described (Sudo et al., 2003; Halls et al., 2005). Co-transfected cells were incubated with increasing concentrations of INSL3 for $6 \mathrm{~h}$ after which the media was aspirated and the cells frozen at $-80^{\circ} \mathrm{C}$ overnight. The amount of cAMP-driven $\beta$-galactosidase expression was then determined in each well as described (Scott et al., 2006). The cAMP activity responses to ligand stimulation were normalized to the maximal cAMP activity response to INSL3. Data points were performed in triplicate and each experiment was repeated three times. Concentration response curves were analyzed using GraphPad PRISM (GraphPad Inc) and a sigmoidal dose-response curve with variable slope model was used to plot curves and calculate $\mathrm{pEC}_{50}$ values. Final pooled pEC $_{50}$ data were analyzed using one-way ANOVA coupled to Newman-Keuls multiple comparison test for multiple group comparison.

\section{RESULTS \\ CHIMERIC PEPTIDES}

The INSL3 B-chain was combined with the A chains of other relaxin family peptides to produce the chimeric peptides $\mathrm{H} 2$ A/INSL3-B, INSL6-A/INSL3-B, and INSL5-A/INSL3-B (Table 1). This strategy has been used previously to demonstrate the key role of the relaxin A-chain in binding and activation of its receptor RXFP1 (Liu et al., 2005). All of the chimeric peptides bound to RXFP2 with high affinity and similar pKi as INSL3 (Table 1, Figure 2A). In contrast the ability of the chimeric peptides to stimulate cAMP activity in RXFP2 receptor expressing cells was severely compromised. INSL6-A/INSL3-B and INSL5-A/INSL3-B were unable to stimulate cAMP in concentrations up to $1 \mu \mathrm{M}$. These chimeric peptides therefore must be able to bind to RXFP2 with high affinity through the INSL3 B-chain but have lost the ability to activate RXFP2 and are high affinity antagonists.

The H2-A/INSL3-B chimera was expected to be able to bind and activate RXFP2 as $\mathrm{H} 2$ relaxin is also a full agonist of RXFP2 (Table 1, Figure 2B). However, while H2-A/INSL3-B was able to bind to RXFP2 with high affinity, and it showed a similar pEC50 to both INSL3 and $\mathrm{H} 2$ relaxin it was only able to stimulate to $45 \%$ of the maximal INSL3 effect demonstrating properties of a partial agonist (Table 1, Figure 2B). We postulated that this probably reflects the fact that native $\mathrm{H} 2$ relaxin binds and activates RXFP2 in a different manner to INSL3. Thus the binding of the INSL3 B-chain to the RXFP2 LRRs may not be compatible with the binding mode of the $\mathrm{H} 2$ relaxin $\mathrm{A}$-chain to the transmembrane domain of RXFP2. To test this hypothesis, we examined the activity of H2-A/INSL3-B chimera on a chimeric receptor RXFP2/1, whereby the ectodomain of RXFP2 is fused to the transmembrane domains of RXFP1. As demonstrated in Figure 3, H2-A/INSL3-B appears to behave, like INSL3 and $\mathrm{H} 2$ relaxin, as a full agonist at this chimeric receptor, i.e., the RXFP1 transmembrane domains are able to rescue the full agonist activity of this peptide.

\section{INSL5-A/INSL3-B PARTIAL CHIMERAS}

The chimeric peptide experiments above indicated that the Achain of INSL3 is responsible for the ability of INSL3 to activate RXFP2. Therefore as a means to determine the A-chain region(s) that mediate the activation by INSL3, we prepared INSL3 peptides in which INSL3 A-chain residues were replaced with those from the INSL5 peptide as the INSL5-A/INSL3-B peptide was completely inactive (Table 1). Importantly, all of the partial chimeras were able to bind to RXFP2 with near native affinity (Table 1, Figure 4A). Replacement of residues within the core of the Achain from amino acid $12-23$ to INSL5 specific residues had no effect on the activity of the chimeric peptides which indicated that residues in this region are not involved with receptor activation (Table 1, Figure 4B). In contrast, replacement of residues 1-9 with the six residues of the INSL5 A-chain N-terminus resulted in a significant loss of peptide activity. We then replaced individual regions of the A-chain $\mathrm{N}$-terminus A5/6 and A8/9 but neither of these substitutions resulted in a loss of peptide activity.

\section{INSL3 A-CHAIN VARIANTS}

The finding that replacement of the entire $\mathrm{N}$-terminal portion of INSL3, residues A1-9, with the N-terminal portion of INSL5 significantly affected activation, but that no effects were seen when smaller segments were changed is intriguing. It is however in agreement with previous data showing that individual replacement of residues in this region with alanines does not have an affect on signaling (Bullesbach and Schwabe, 2005a). To further investigate we replaced the entire region with either four or five consecutive alanine residues to create Ala-4 A(10-26) INSL3 and Ala-5 A(10-26) INSL3. Both of these peptides bound to RXFP2 with near native affinity (Table 2, Figure 5A) and, in contrast to the variants carrying the INSL5 N-terminal portion of the A-chain, were also able to act as full agonists albeit with slightly lower potency than the native peptide ( $p<0.05$ compared to INSL3, Table 2 , Figure $5 \mathrm{~B}$ ). Therefore it is clear that the actual residues in these positions are not important for the ability of INSL3 to activate its receptor.

Additionally, we investigated the role of the two residues at the A-chain C-terminus. INSL3 peptides from all species contain two amino acids in this position (Wilkinson et al., 2005) but the effect of removal of these residues on peptide activity has not been tested. Although INSL3 $\Delta 25 / 26$ demonstrated binding affinity comparative to that of INSL3 (Table 2, Figure 6A), it showed significantly reduced ability to stimulate cAMP compared to the native peptide $(p<0.01$, Table 2, Figure 6B). The A-chain C-terminus therefore 
Table 1 | Sequences and receptor binding affinities (pKi) and receptor activation (pEC50) activities of INSL3, H2 relaxin, and INSL3 chimeric peptides.

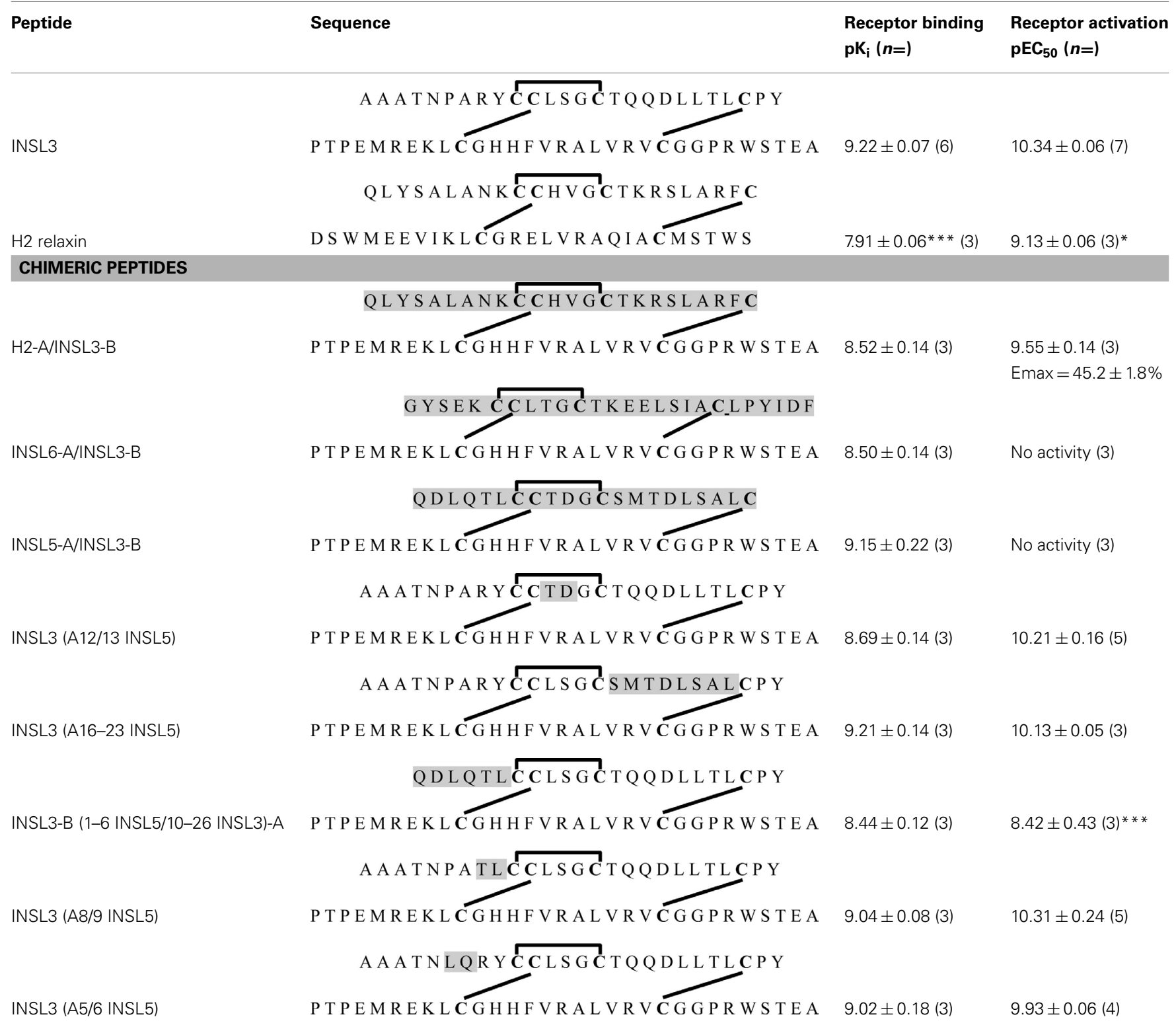

${ }^{*} p<0.05,{ }^{* * *} p<0.001 \mathrm{vs}$. INSL3. Shaded amino acids are those which have been changed to INSL5 residues in the chimeric peptides.

appears to play at least a small role in the ability of INSL3 to activate its receptor.

\section{INSL3 B-CHAIN VARIANTS}

Previous studies have also suggested that specific residues in the Bchain N-terminus may also contribute to the activity of the INSL3 peptide (Bullesbach and Schwabe, 2007). To confirm these findings we synthesized two INSL3 B-chain mutants to assess their activity in our cell systems. GluB7 to alanine did not show any affect on either binding or activation, consistent with previous findings (Bullesbach and Schwabe, 2006). However, contrary to a previous report (Bullesbach and Schwabe, 2006) mutation of LysB8 to alanine did result in the loss of peptide activity $(p<0.05)$ without any effect on receptor binding (Table 3, Figures 6A,B). This result is not likely to be due to differences in cell line or signaling assay used as the authors also measured cAMP signaling in transfected HEK-293T cells (Bullesbach and Schwabe, 2006). It is therefore possible that this residue does play a role in receptor activation.

\section{TRUNCATED INSL3 ANALOGS}

Finally based on the knowledge of the role of specific residues in the A-chain and B-chain of INSL3 that are important for binding and activity, we postulated truncated INSL3 analogs that would retain most of the native activity. We therefore synthesized INSL3 A(5-26)/B(1-27) and tested its ability to bind and activate RXFP2. This peptide retained high affinity binding and was able 

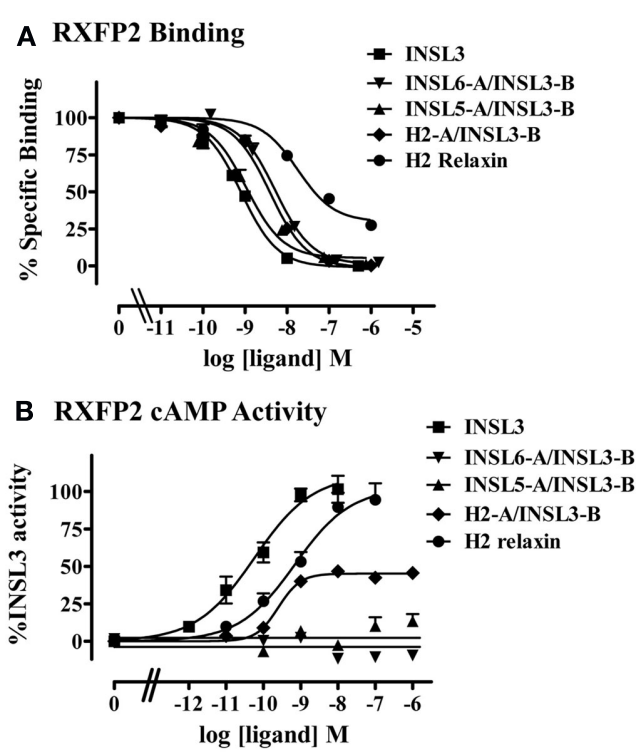

FIGURE 2 | Activity of the INSL3 chimeric peptides on the RXFP2 receptor. (A) Competition binding utilizing Eu-labeled INSL3 compared to INSL3 and $\mathrm{H} 2$ relaxin. Data are expressed as the percentage of specific binding and are pooled from at least three experiments with triplicate determinations within each assay. (B) CAMP activity compared to INSL3 and $\mathrm{H} 2$ relaxin measured utilizing a $\mathrm{pCRE}-\beta$-galactosidase reporter gene assay. Data are expressed as the percentage INSL3 maximum activity and are pooled data from at least three experiments with triplicate determinations within each assay.

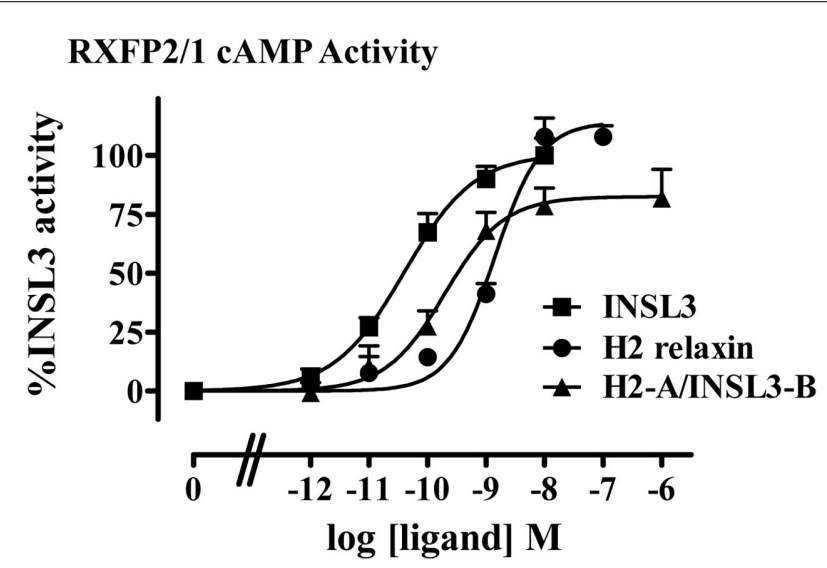

FIGURE 3 | cAMP activity of H2-A/INSL3-B compared to INSL3 and H2 relaxin at the RXFP2/1 chimeric receptor measured utilizing a pCRE- $\boldsymbol{\beta}$-galactosidase reporter gene assay. Data are expressed as the percentage INSL3 maximum activity and are pooled data from at least three experiments with triplicate determinations within each assay.

to activate cAMP with only slightly reduced potency compared to native INSL3 (Table 3, Figures 7A,B). We therefore synthesized INSL3 A(5-26)/B(7-27) which was also demonstrated to retain high affinity binding and also retained similar potency compared to native INSL3 (Table 3, Figures 7A,B). We have therefore been able to produce a truncated INSL3 peptide that possesses 14 fewer

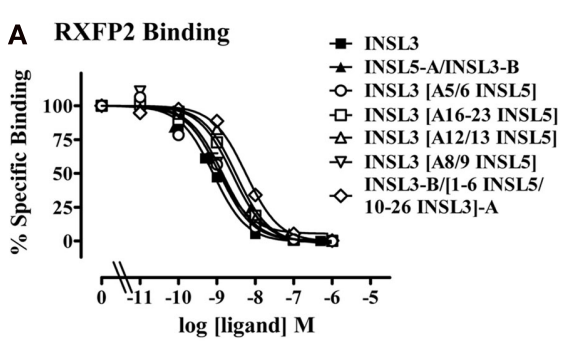

B RXFP2 cAMP Activity

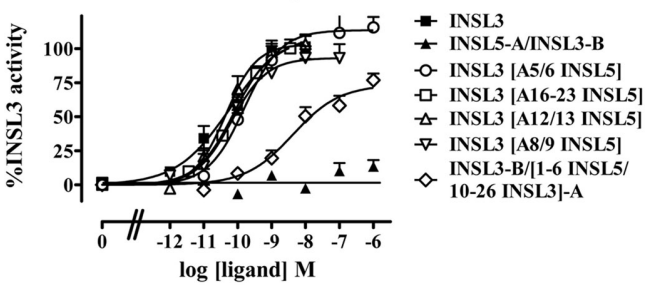

FIGURE 4 | Activity of the INSL5-A/INSL3-B partial chimeric peptides on the RXFP2 receptor. (A) Competition binding utilizing Eu-labeled INSL3 compared to INSL3 and H2 relaxin. Data are expressed as the percentage of specific binding and are pooled from at least three experiments with triplicate determinations within each assay. (B) CAMP activity compared to INSL3 and $\mathrm{H} 2$ relaxin measured utilizing a $\mathrm{pCRE}-\beta$-galactosidase reporter gene assay. Data are expressed as the percentage INSL3 maximum activity and are pooled data from at least three experiments with triplicate determinations within each assay.

residues than the native peptide, i.e., $25 \%$ smaller and retains near native activity.

\section{DISCUSSION}

The binding of ligand to RXFP1 and RXFP2 and subsequent structural rearrangements that lead to intracellular signaling through these receptors is a complex process (Bathgate et al., 2006a; Scott et al., 2006). Although the significant efforts directed at understanding the ligand mediated activation of these receptors over recent years have led to a number of new insights, in particular a detailed picture of the interaction between the hormone B-chain and the receptor LRR domain, still very little is known about how the receptors are activated. This study was designed to shed further light on the activation of RXFP2 by INSL3.

\section{INSL3 CHIMERAS CONFIRM THE ROLE OF THE INSL3 A-CHAIN IN RECEPTOR ACTIVATION}

Given the two-chain nature of the relaxin peptides creation of chimeric ligands comprising an A- and a B-chain from different relaxins offers a convenient way to decipher the role of different domains in binding and activation of the relaxin receptors. Chimeric variants of the related relaxin-3 peptide were instrumental in decoding the key role of the A-chain in the ability of relaxin-3 to activate RXFP1, while also showing that the A-chain is not necessary for activation of its endogenous receptor RXFP3 (Liu et al., 2005). This observation led to the subsequent design of peptides that selectively target RXFP3 (Liu et al., 2005; Kuei et al., 2007; Haugaard-Kedstrom et al., 2011). Structural studies using NMR spectroscopy revealed minimal overall structural effect 
Table 2 | Sequences and receptor binding affinities (pKi) and receptor activation (pEC50) activities of INSL3 and INSL3 A chain variants.

\begin{tabular}{|c|c|c|c|}
\hline Peptide & Sequence & $\begin{array}{l}\text { Receptor binding } \\
\mathbf{p K}_{\mathbf{i}}(n=)\end{array}$ & $\begin{array}{l}\text { Receptor activation } \\
\text { pEC }_{50}(n=)\end{array}$ \\
\hline INSL3 & P T P E M R E K L CGH H F V R A L V R V CG G P R S T E A & $9.22 \pm 0.07(6)$ & $10.34 \pm 0.06(7)$ \\
\hline \multicolumn{4}{|c|}{ INSL3 A CHAIN VARIANTS } \\
\hline Ala-5 A(10-26) INSL3 & P T P E M R E K L C & $8.80 \pm 0.13(3)$ & $9.51 \pm 0.08(3)^{*}$ \\
\hline
\end{tabular}

${ }^{*} p<0.05,{ }^{*} p<0.01$ vs. INSL3. Shaded area is the alanine residues that have been added to the truncated INSL3 peptide.

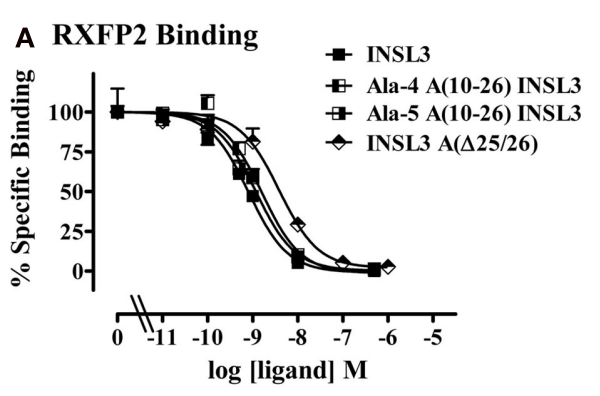

B RXFP2 cAMP Activity

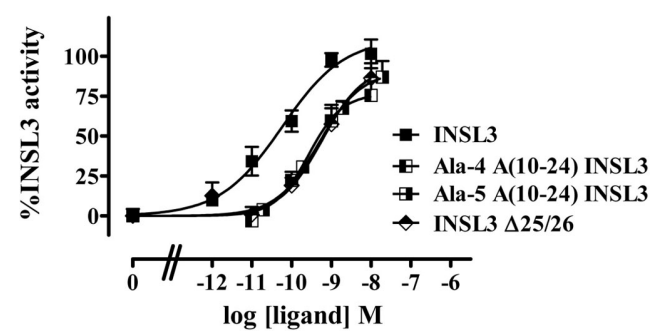

FIGURE 5 | Activity of INSL3 A-chain variant peptides on the RXFP2 receptor. (A) Competition binding utilizing Eu-labeled INSL3 compared to INSL3 and $\mathrm{H} 2$ relaxin. Data are expressed as the percentage of specific binding and are pooled from at least three experiments with triplicate determinations within each assay. (B) CAMP activity compared to INSL3 and $\mathrm{H} 2$ relaxin measured utilizing a $\mathrm{pCRE}-\beta$-galactosidase reporter gene assay. Data are expressed as the percentage INSL3 maximum activity and are pooled data from at least three experiments with triplicate determinations within each assay.

when combining the relaxin-3 B-chain with the INSL5 A-chain, suggesting that relaxin chains are generally interchangeable without large conformational effects (Haugaard-Jonsson et al., 2008). Here we generated a series of chimeric peptides based on the INSL3 B-chain, which is known to carry the primary receptor

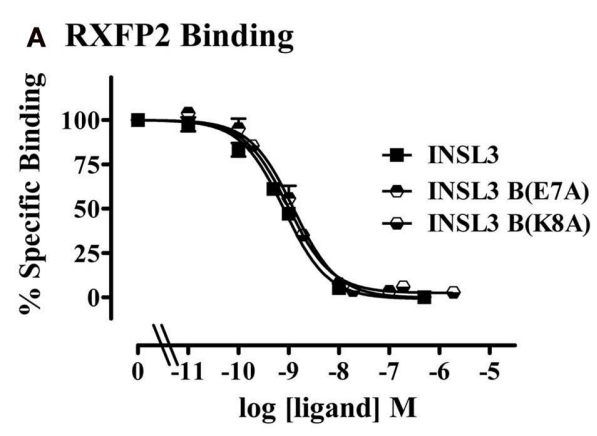

\section{B RXFP2 cAMP Activity}

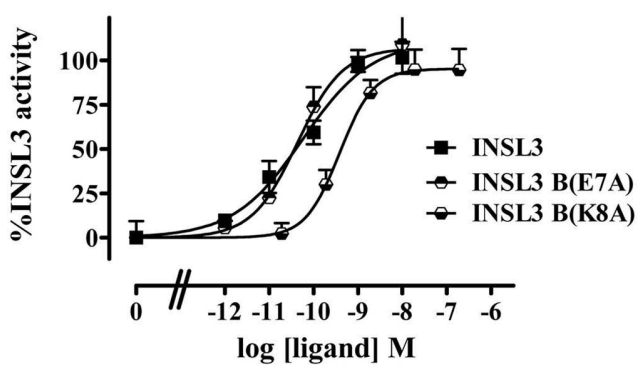

FIGURE 6 | Activity of INSL3 B-chain variant peptides on the RXFP2 receptor. (A) Competition binding utilizing Eu-labeled INSL3 compared to INSL3 and $\mathrm{H} 2$ relaxin. Data are expressed as the percentage of specific binding and are pooled from at least three experiments with triplicate determinations within each assay. (B) CAMP activity compared to INSL3 and $\mathrm{H} 2$ relaxin measured utilizing a $\mathrm{pCRE}-\beta$-galactosidase reporter gene assay. Data are expressed as the percentage INSL3 maximum activity and are pooled data from at least three experiments with triplicate determinations within each assay.

binding site directed toward the RXFP2 LRRs (Bullesbach and Schwabe, 2006; Rosengren et al., 2006; Scott et al., 2007). Strikingly all chimeric peptides displayed negligible change of RXFP2 
Table 3 | Sequences and receptor binding affinities (pKi) and receptor activation (pEC50) activities of INSL3, INSL3 B chain variants, and truncated INSL3 analogs.

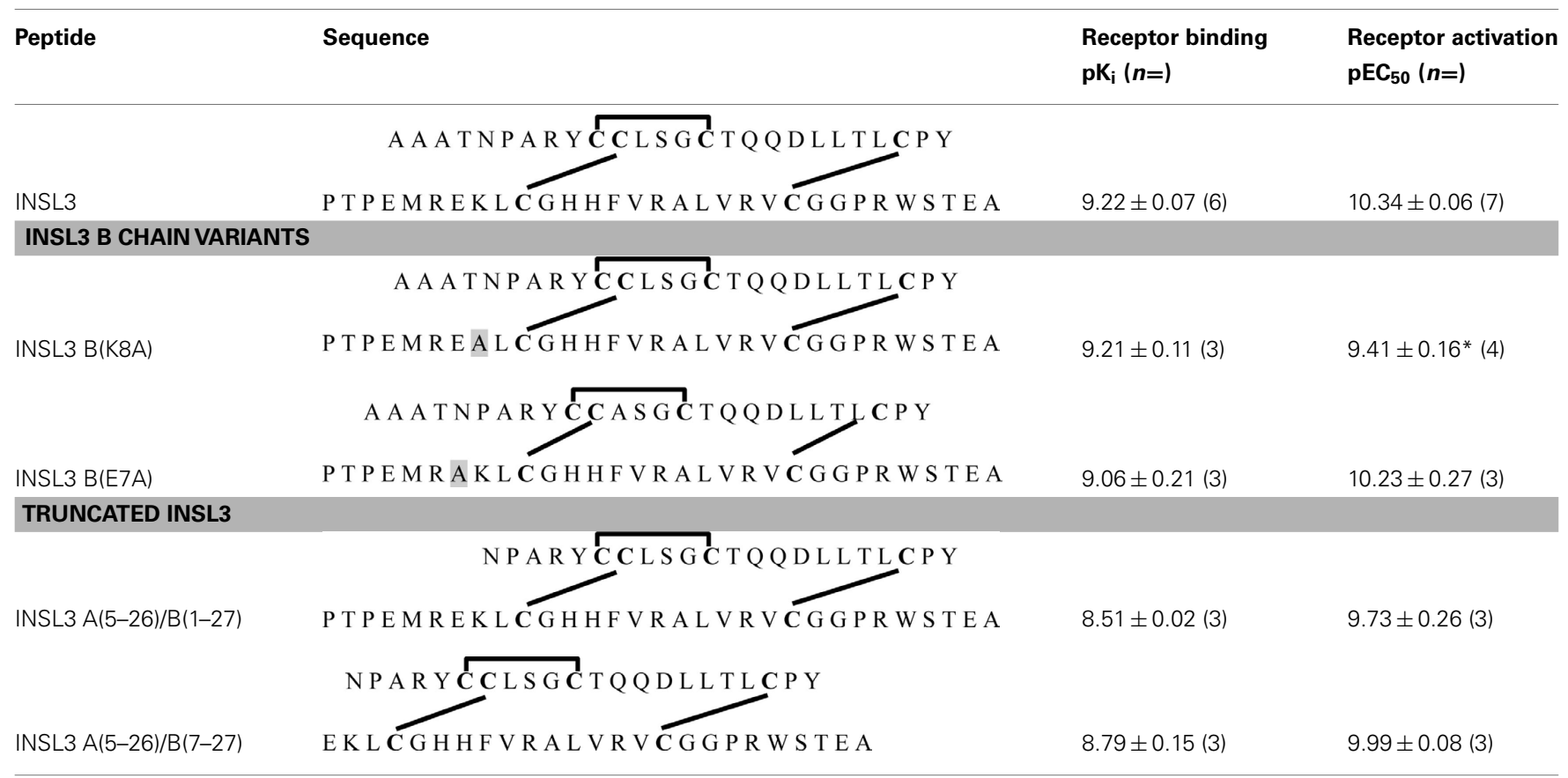

${ }^{*} p<0.05 \mathrm{vs}$. INSL3. The shading highlights the amino acid which has been changed to alanine in the mutant peptides INSL3 B(K8A) and B(E7A).

binding but analogs carrying A-chains from INSL5 or INSL6 were unable to induce any receptor signaling, highlighting the role of the INSL3 A-chain in the activation of RXFP2. In contrast, when the $\mathrm{H} 2$ relaxin A-chain was combined with the INSL3 B-chain, a molecule was obtained that activated RXFP2 with an EC50 similar to native INSL3. However, this compound has an Emax of only $45 \%$ revealing that this peptide is only a partial agonist. This finding is particularly interesting given that $\mathrm{H} 2$ relaxin itself is a full agonist for RXFP2.

We postulated that this was probably due to $\mathrm{H} 2$ relaxin binding and activating RXFP2 in a different manner to INSL3. Hence the mode by which the INSL3 B-chain binds to the RXFP2 LRR domain is not fully compatible with the interaction between the $\mathrm{H} 2$ relaxin A-chain and the RXFP2 extracellular loops necessary for full receptor activation. To confirm this idea, the chimeric peptide was tested against a chimeric receptor comprising the LRR domain of RXFP2 attached to the transmembrane domain of the $\mathrm{H} 2$ relaxin receptor RXFP1 (Sudo et al., 2003). In this system full activation of the receptor was observed, clearly demonstrating that despite the structural similarities of both $\mathrm{H} 2$ relaxin/INSL3 and their receptors RXFP1/RXFP2 the mechanism whereby the peptides activate their receptors is distinctly different. Importantly the mechanism of activation appears to be guided by the peptide rather than the receptor, i.e., $\mathrm{H} 2$ relaxin activates $\mathrm{RXFP} 2$ in a similar way as it actives RXFP1 rather than the way INSL3 activates RXFP2. This is consistent with previous data showing that truncation of the $\mathrm{H} 2$ relaxin $\mathrm{A}$-chain had differing effects on the binding and activation of RXFP1 and RXFP2 (Hossain et al., 2008).

To further narrow down the regions of importance in the INSL3 A-chain a series of partial A-chain chimeras of INSL3 and
INSL5 were created. Although introduction of residues from the A-chain of INSL5 in the region 12-23 had little or no effect on the potency of the peptide, replacement of residues A1-9 with residues A1-6 of INSL5 resulted in a significant decrease in activation of RXFP2. This finding is consistent with previous truncation studies demonstrating that residues A1-5 of INSL3 can be truncated without detrimental effect, but that further truncations lead to a decrease in receptor activation; indeed, a peptide lacking the first eight residues is a high affinity antagonist (Bullesbach and Schwabe, 2005a, 2007). It is interesting that the introduction of only two residues from INSL5 at a time (i.e., A5/6 or A8/9), had no effect on receptor activation. This was consistent with previous studies which demonstrated that mutations of A8/9 to alanine had no effect on INSL3 activity (Bullesbach and Schwabe, 2006).

\section{ALANINE SUBSTITUTIONS REVEAL THAT NO INDIVIDUAL RESIDUES IN THE A-CHAIN ARE CRITICAL FOR ACTIVITY}

In light of our findings that replacement of the entire $\mathrm{N}$-terminal region but not smaller segments affected receptor activation, and the previously published data in which replacement of individual residues with alanines were found not to compromise activation (Bullesbach and Schwabe, 2006), we created two analogs comprising strings of alanines preceding CysA10. This region adopts a helix in native INSL3; one would expect that the added alanine residues would be able to retain the native structure, but they would do so without presenting any functionally relevant side chains. Indeed both Ala5- and Ala4-(A10-26) INSL3 were able to activate RXFP2, indicating that an "inert" peptide backbone in this region of INSL3 is sufficient for receptor activation. 


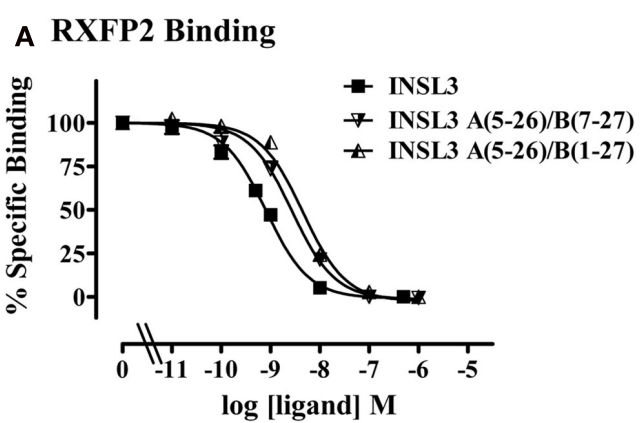

B RXFP2 cAMP Activity

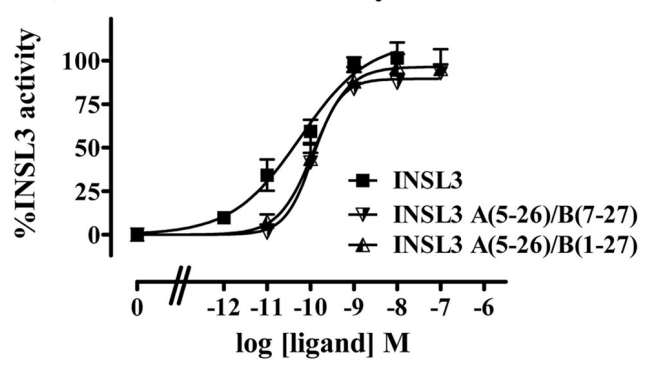

FIGURE 7 | Activity of INSL3 truncated peptides on the RXFP2 receptor. (A) Competition binding utilizing Eu-labeled INSL3 compared to INSL3 and $\mathrm{H} 2$ relaxin. Data are expressed as the percentage of specific binding and are pooled from at least three experiments with triplicate determinations within each assay. (B) CAMP activity compared to INSL3 and $\mathrm{H} 2$ relaxin measured utilizing a $\mathrm{pCRE}-\beta$-galactosidase reporter gene assay. Data are expressed as the percentage INSL3 maximum activity and are pooled data from at least three experiments with triplicate determinations within each assay.

\section{WHAT IS THE MECHANISM OF ACTIVATION OF RXFP2 BY INSL3?}

Our data and previous studies firmly establish the region of residues A6-A9 in INSL3 as the key domain for receptor activation (Bullesbach and Schwabe, 2005a, 2006, 2007). However, it is striking that neither previous studies nor our work here have been able to identify any significant contributions from the individual side chains present in this region. In fact replacing the entire $\mathrm{N}$ terminal segment with a series of alanines is sufficient for retaining receptor activation. It has been suggested that rather than contributions from side chain groups the key drivers of the interaction may be hydrogen bonding interactions involving the polar groups of the peptide backbone (Bullesbach and Schwabe, 2007). Based on the multi-alanine versions presented here this appears to be the only logical explanation, but if the nature of the residues in this region is irrelevant, why is the peptide carrying the $\mathrm{N}$ terminal portion of the INSL5 A-chain unable to fully activate the receptor? The answer may be that a particular structure in this region is required for the correct orientation of the backbone groups for the interaction with the receptor. The NMR solution structure of native INSL3 reveals that the $\mathrm{N}$-terminal region, like insulin and other relaxin peptides, adopts a helical conformation. In INSL3 the helical region spans residues A6-A12, while the Nterminal five residues are disordered (Rosengren et al., 2006). Should this structure be retained upon receptor binding then the amide protons of AlaA7 and ArgA8, which are not involved in inter-helical hydrogen bonds and constitute the positive part of the helix dipole would be prime candidates for interacting with the receptor. Bullesbach and Schwabe (2007) showed that $N$-methylating ArgA8 or TyrA9 and thereby removing their hydrogen bonding ability attenuates receptor activation, which would be consistent with such an interaction. In the NMR structure of INSL5 the A-chain N-terminal helix extends further towards the $\mathrm{N}$-terminus, likely because the N-terminal residue is a pyroglutamic acid removing the clash between the $\mathrm{N}$-terminal positive charge and the helix dipole; the helix is further stabilized by the presence of an Asp at position A2, which caps the N-terminal part of the helix (Haugaard-Jonsson et al., 2009). These features may explain the inability of the INSL5 A-chain to activate RXFP2 in the chimeric peptides. We cannot of course rule out the possibility that it is a requirement for activation that the structure in this region is altered upon binding to RXFP2, and that by introducing a more stable helix, this structural rearrangement is prevented. Such an event could expose further hydrogen bond donors and acceptors of the peptide backbone for interaction with the receptor. The N-terminal region of INSL3 is somewhat dynamic as evident from amide exchange rates and line broadening observed in NMR studies, and this dynamic character may be relevant for function (Rosengren et al., 2006), but the nature of what an altered receptor bound form might be is difficult to predict.

\section{DO OTHER REGIONS OF INSL3 CONTRIBUTE TO THE ACTIVATION DOMAIN?}

Bullesbach and Schwabe (2005a, 2007) demonstrated that truncation of up to five residues of the B-chain $\mathrm{N}$-terminus does not affect binding or signaling, but that further truncations result in a step wise decrease in both binding and activation. Again this contribution was speculated to be independent of the nature of the side chains in this region, as replacement of either GluB7 or LysB8 with alanine did not appear to affect receptor binding or activation. We decided to revisit these findings and synthesized these two analogs. Consistent with previous data the GluB7 to alanine analog showed native-like binding and activation. However, in contrast to the previous report we found that the substitution in INSL3 of LysB8 with alanine caused a significant drop in activation of RXFP2 with no change in binding affinity. Hence, unlike substitution of other B-chain residues where loss of activity is associated with loss of binding, LysB8 appears to be involved in activation only. An analysis of the INSL3 NMR structure reveals that LysB8 is tightly associated with the C-terminal part of the A-chain N-terminal helix, providing a capping effect by interacting with the carbonyls of CysA11, LeuA12, and SerA13. This interaction with the negative part of the helix dipole is likely to have a significant stabilizing effect on the helix and thus the effects of severely truncating or mutating the B-chain $\mathrm{N}$-terminal region on RXFP2 receptor activation may be related to a secondary structural effect destabilizing the A-chain, rather than direct contacts with the receptor. Here we also demonstrated that truncation of the C-terminal two residues of the INSL3 Achain decreased activity. These residues are rather distant from the key $\mathrm{N}$-terminal region of the A-chain and it would seem unlikely that they form part of the same receptor interaction 
responsible for activation. More likely is that this observation is related to disruption of contacts between the two A-chain helices or to interference with the spatial coordination of the activation domain in the A-chain with the key LRR primary binding site in the B-chain, which is in close contact with ProA25 and TyrA26.

\section{MINIMAL ACTIVE STRUCTURE OF INSL3}

Finally, utilizing the data gained in this study, and from previous studies, we designed INSL3 peptides that should represent active truncated INSL3 analogs. We were able to show that INSL3 $\mathrm{A}(5-26) / \mathrm{B}(7-27)$ retained near native activity. This peptide is $25 \%$ smaller than the native peptide and thus easier and cheaper to make and represents a potential lead peptide for further development of

\section{REFERENCES}

Adham, I. M., Burkhardt, E., Benahmed, M., and Engel, W. (1993). Cloning of a cDNA for a novel insulin-like peptide of the testicular Leydig cells. J. Biol. Chem. 268, 26668-26672.

Bamberger, A. M., Ivell, R., Balvers, M., Kelp, B., Bamberger, C. M., Riethdorf, L., and Loning, T. (1999). Relaxin-like factor (RLF): a new specific marker for Leydig cells in the ovary. Int. J. Gynecol. Pathol. 18, 163-168.

Bathgate, R., Balvers, M., Hunt, N., and Ivell, R. (1996). Relaxin-like factor gene is highly expressed in the bovine ovary of the cycle and pregnancy: sequence and messenger ribonucleic acid analysis. Biol. Reprod. 55, 1452-1457.

Bathgate, R. A., Ivell, R., Sanborn, B. M., Sherwood, O. D., and Summers, R. J. (2006a). International Union of Pharmacology: recommendations for the nomenclature of receptors for relaxin family peptides. Pharmacol. Rev. 58, 7-31.

Bathgate, R. A. D., Hsueh, A. J., and Sherwood, O. D. (2006b). "Physiology and molecular biology of the relaxin peptide family," in Physiology of Reproduction, ed. J. D. Neill III (San Diego: Elsevier), 679-770.

Bullesbach, E. E., and Schwabe, C. (1995). A novel Leydig cell cDNA-derived protein is a relaxinlike factor. J. Biol. Chem. 270, 16011-16015.

Bullesbach, E. E., and Schwabe, C. (2005a). LGR8 signal activation by the relaxin-like factor. J. Biol. Chem. 280, 14586-14590.

Bullesbach, E. E., and Schwabe, C. (2005b). The trap-like relaxinbinding site of the leucine-rich Gprotein-coupled receptor 7. J. Biol. Chem. 280, 14051-14056.

Bullesbach, E. E., and Schwabe, C. (2006). The mode of interaction of the relaxin-like factor (RLF) with the leucine-rich repeat $G$ proteinactivated receptor 8. J. Biol. Chem. 281, 26136-26143.

Bullesbach, E. E., and Schwabe, C. (2007). Structure of the transmembrane signal initiation site of the relaxin-like factor (RLF/INSL3). Biochemistry 46, 9722-9727.

Chen, W., Shields, T. S., Stork, P. J., and Cone, R. D. (1995). A colorimetric assay for measuring activation of Gsand $\mathrm{Gq}$-coupled signaling pathways.

Gorlov, I. P., Kamat, A., Bogatcheva, N. V., Jones, E., Lamb, D. J., Truong, A., Bishop, C. E., Mcelreavey, K., and Agoulnik, A. I. (2002). Mutations of the GREAT gene cause cryptorchidism. Hum. Mol. Genet. 11, 2309-2318.

Halls, M. L., Bond, C. P., Sudo, S., Kumagai, J., Ferraro, T., Layfield, S., Bathgate, R. A., and Summers, R. J. (2005). Multiple binding sites revealed by interaction of relaxin family peptides with native and chimeric relaxin family peptide receptors 1 and 2 (LGR7 and LGR8). J. Pharmacol. Exp. Ther. 313, 677-687.

Hartley, B. J., Scott, D. J., Callander, G. E., Wilkinson, T. N., Ganella, D. E., Kong, C. K., Layfield, S., Ferraro, T., Petrie, E. J., and Bathgate, R. a. D. (2009). Resolving the unconventional mechanisms underlying RXFP1 and RXFP2 receptor function. Ann. N. Y. Acad. Sci. 1160, 67-73.

Haugaard-Jonsson, L. M., Hossain, M. A., Daly, N. L., Bathgate, R. A., Wade, J. D., Craik, D. J., and Rosengren, K. J. (2008). Structure of the R3/I5 Chimeric Relaxin Peptide, a Selective GPCR135 and GPCR142 Agonist. J. Biol. Chem. 283, 23811-23818.

Haugaard-Jonsson, L. M., Hossain, M. A., Daly, N. L., Craik, D. J., Wade, J. D., and Rosengren, K. J. Anal. Biochem. 226, 349-354.

smaller INSL3 analogs that may have great therapeutic potential as fertility regulators.

\section{ACKNOWLEDGMENTS}

The authors would like to thank Tania Ferraro and Sharon Layfield for binding and signaling assays, Dr. Satoko Sudo and Prof Aaron Hsueh for provision of the RXFP2/1 construct, and Prof. Geoff Tregear for support. Studies at the FNI were supported by NHMRC grants 509048 and 628427 and by the Victorian Government's Operational Infrastructure Support Program. Ross A. D. Bathgate and John D. Wade are recipients of Australian National Health and Medical Research Council (NHMRC) Research Fellowships. K. Johan Rosengren is a recipient of a NHMRC Career Development Award.

(2009). Structure of human insulinlike peptide 5 and characterization of conserved hydrogen bonds and electrostatic interactions within the relaxin framework. Biochem. J. 419, 619-627.

Haugaard-Kedstrom, L. M., Shabanpoor, F., Hossain, M. A., Clark, R. J., Ryan, P. J., Craik, D. J., Gundlach, A. L., Wade, J. D., Bathgate, R. A., and Rosengren, K. J. (2011). Design, synthesis, and characterization of a single-chain peptide antagonist for the relaxin-3 receptor RXFP3. J. Am. Chem. Soc. 133, 4965-4974.

Hossain, M. A., Rosengren, K. J., Haugaard-Jonsson, L. M., Zhang, S., Layfield, S., Ferraro, T., Daly, N. L., Tregear, G. W., Wade, J. D., and Bathgate, R. A. (2008). The A-chain of human relaxin family peptides has distinct roles in the binding and activation of the different relaxin family peptide receptors. J. Biol. Chem. 283, 17287-17297.

Hsu, S. Y., Nakabayashi, K., Nishi, S., Kumagai, J., Kudo, M., Sherwood, O. D., and Hsueh, A. J. (2002). Activation of orphan receptors by the hormone relaxin. Science 295, 671-674.

Irving-Rodgers, H. F., Bathgate, R. A., Ivell, R., Domagalski, R., and Rodgers, R. J. (2002). Dynamic changes in the expression of relaxinlike factor (INSL3), cholesterol sidechain cleavage cytochrome p450, and 3beta-hydroxysteroid dehydrogenase in bovine ovarian follicles during growth and atresia. Biol. Reprod. 66, 934-943.

Ivell, R., and Bathgate, R. A. (2002). Reproductive biology of the relaxinlike factor (RLF/INSL3). Biol. Reprod. 67, 699-705.

Kawamura, K., Kumagai, J., Sudo, S., Chun, S. Y., Pisarska, M., Morita, H., Toppari, J., Fu, P., Wade, J. D., Bathgate, R. A., and Hsueh, A. J. (2004). Paracrine regulation of mammalian oocyte maturation and male germ cell survival. Proc. Natl. Acad. Sci. U.S.A. 101, 7323-7328.

Kuei, C., Sutton, S., Bonaventure, P., Pudiak, C., Shelton, J., Zhu, J., Nepomuceno, D., Wu, J., Chen, J., Kamme, F., Seierstad, M., Hack, M. D., Bathgate, R. A., Hossain, M. A., Wade J. D., Atack, J., Lovenberg, T. W., and Liu, C. (2007). R3(BDelta23 27)R/I5 chimeric peptide, a selective antagonist for GPCR135 and GPCR142 over relaxin receptor LGR7: in vitro and in vivo characterization. J. Biol. Chem. 282, 25425-25435.

Kumagai, J., Hsu, S. Y., Matsumi, H., Roh, J. S., Fu, P., Wade, J. D., Bathgate, R. A., and Hsueh, A. J. (2002). INSL3/Leydig insulin-like peptide activates the LGR8 receptor important in testis descent. J. Biol. Chem. 277, 31283-31286.

Liu, C., Chen, J., Kuei, C., Sutton, S., Nepomuceno, D., Bonaventure, P., and Lovenberg, T. W. (2005). Relaxin-3/insulin-like peptide 5 chimeric peptide, a selective ligand for $G$ protein-coupled receptor (GPCR)135 and GPCR142 over leucine-rich repeat-containing G protein-coupled receptor 7. Mol. Pharmacol. 67, 231-240.

Nef, S., and Parada, L. F. (1999). Cryptorchidism in mice mutant for Insl3. Nat. Genet. 22, 295-299.

Rosengren, K. J., Bathgate, R. A., Craik, D. J., Daly, N. L., Haugaard-Jonsson, L. M., Hossain, M. A., and Wade, J. D. (2009). Structural insights into the function of relaxins. Ann. N. Y. Acad. Sci. 1160, 20-26.

Rosengren, K. J., Zhang, S., Lin, F., Daly, N. L., Scott, D. J., Hughes R. A., Bathgate, R. A., Craik, D. J., and Wade, J. D. (2006). Solution structure and characterization of the receptor binding surface of insulinlike peptide 3. J. Biol. Chem. 38, 28287-28295. 
Scott, D. J., Layfield, S., Yan, Y., Sudo, S., Hsueh, A. J., Tregear, G. W., and Bathgate, R. A. (2006). Characterization of novel splice variants of LGR7 and LGR8 reveals that receptor signaling is mediated by their unique LDLa modules. J. Biol. Chem. 281, 34942-34954.

Scott, D. J., Tregear, G. W., and Bathgate, R. a. D. (2009). Modelling the primary binding site of RXFP1 and RXFP2. Ann. N. Y. Acad. Sci. 1160, 74-77.

Scott, D. J., Wilkinson, T. N., Zhang, S., Ferraro, T., Wade, J. D., Tregear, G. W., and Bathgate, R. A. (2007). Defining the LGR8 residues involved in binding insulin-like peptide 3 . Mol. Endocrinol. 21, 1699-1712.

Shabanpoor, F., Hughes, R. A., Bathgate, R. A., Zhang, S., Scanlon, D. B., Lin, F., Hossain, M. A., Separovic, F., and Wade, J. D. (2008). Solidphase synthesis of europium-labeled human INSL3 as a novel probe for the study of ligand-receptor interactions. Bioconjug. Chem. 19, 1456-1463.

Spanel-Borowski, K., Schafer, I., Zimmermann, S., Engel, W., and Adham, I. M. (2001). Increase in final stages of follicular atresia and premature decay of corpora lutea in Insl3deficient mice. Mol. Reprod. Dev. 58, 281-286.

Sudo, S., Kumagai, J., Nishi, S., Layfield, S., Ferraro, T., Bathgate, R. A., and Hsueh, A. J. (2003). H3 relaxin is a specific ligand for LGR7 and activates the receptor by interacting with both the ectodomain and the exoloop 2. J. Biol. Chem. 278, 7855-7862.

Tashima, L. S., Hieber, A. D., Greenwood, F. C., and Bryant-Greenwood, G. D. (1995). The human Leydig insulin-like (hLEY I-L) gene is expressed in the corpus luteum and trophoblast. J. Clin. Endocrinol. Metab. 80, 707-710.
Wilkinson, T. N., Speed, T. P., Tregear, G. W., and Bathgate, R. a. D. (2005). Evolution of the relaxin-like peptide family. BMC Evol. Biol. 5, 14. doi:10.1186/1471-2148-5-14

Zhang, S., Hughes, R. A., Bathgate, R. A., Shabanpoor, F., Hossain, M. A., Lin, F., Van Lierop, B., Robinson, A. J., and Wade, J. D. (2010). Role of the intraA-chain disulfide bond of insulinlike peptide 3 in binding and activation of its receptor, RXFP2. Peptides 31, 1730-1736.

Zimmermann, S., Steding, G., Emmen, J. M., Brinkmann, A. O., Nayernia, K., Holstein, A. F., Engel, W., and Adham, I. M. (1999). Targeted disruption of the Insl3 gene causes bilateral cryptorchidism. Mol. Endocrinol. 13, 681-691.

Conflict of Interest Statement: The authors declare that the research was conducted in the absence of any commercial or financial relationships that could be construed as a potential conflict of interest.

Received: 16 December 2011; paperpending published: 10 January 2012; accepted: 12 January 2012; published online: 01 February 2012.

Citation: Bathgate RAD, Zhang S, Hughes RA, Rosengren KJ and Wade JD (2012) The structural determinants of insulin-like peptide 3 activity. Front. Endocrin. 3:11. doi: 10.3389/fendo.2012.00011

This article was submitted to Frontiers in Molecular and Structural Endocrinology, a specialty of Frontiers in Endocrinology. Copyright (C) 2012 Bathgate, Zhang, Hughes, Rosengren and Wade. This is an open-access article distributed under the terms of the Creative Commons Attribution Non Commercial License, which permits non-commercial use, distribution, and reproduction in other forums, provided the original authors and source are credited. 